\title{
Removal of Typical Pharmaceutically Active Compounds in Sewage Sludge Using Anaerobic Digestion Processes
}

\author{
Xuelian Wang, Meng Wang, Haidong Zhou *, Jianbo Liu, Jiaoyan Zhou \\ School of Environment and Architecture \\ University of Shanghai for Science and Technology \\ Shanghai, China \\ E-mail: zhouhaidong@usst.edu.cn
}

\begin{abstract}
The removal of four pharmaceutically active compounds (PhACs), i.e. anti-inflammatory painkiller diclofenac (DFC), lipid regulating agent clofibric acid (CFA), epilepsy drugs carbamazepine (CBM), and broad-spectrum antibacterial agent triclosan (TCS), present in sewage sludge was investigated using anaerobic digestion (AD) processes in the mesophilic and thermophilic mode. Sludge retention times (SRTs) were $10 \mathrm{~d}, 15 \mathrm{~d}$, and $20 \mathrm{~d}$ for the mesophilic mode and 7 d, $15 \mathrm{~d}$, and $20 \mathrm{~d}$ for the thermophilic mode. The effective isolation and purification pretreatment to extract the target compounds from the sewage sludge samples was firstly established, followed by gas chromatography-mass spectrometer (GC-MS) analysis to identify and quantify them. The removal efficiencies of the target compounds could be raised to a certain extent with the increase on SRTs, especially under the mesophilic condition. The removal of CFA and TCS under thermophilic condition hardly varied when the SRTs were above $15 \mathrm{~d}$. All the compounds could be partly removed from the sewage sludge under the two temperature conditions, particularly the triclosan which was obtained about $74 \%$ of removal. Besides, CFA, firstly reported in this study, could obtained maximal $65 \%$ removal efficiency during ADs of sewage sludge. On the whole, the thermophilic mode was more conducive to the removal of CFA, CBM and TCS, but DFC could be better removed in the mesophilic mode.
\end{abstract}

Keywords-removal efficiency; sludge retention time; mesophilic anaerobic digestion; ammonia nitrogen

\section{INTRODUCTION}

Pharmaceutically active compounds (PhACs) represent an overgrowing portion of organic micro-pollutants of the environment, and are increasingly concerned. PhACs in the effluent sewage have been extensively investigated and detected [1]. However, PhACs can absorb onto bacterial lipid structure and fat fraction of the sewage sludge through hydrophobic interactions (e.g., aliphatic and aromatic groups) [2], therefore, the concerns and researches on their concentrations, behavior and fate in the sewage sludge are growing.

Anaerobic digestion (AD) has been extensively used and proven to be the most efficient treatment technology to stabilize organic substrates in sewage sludge [3]. The mesophilic AD is most widely adopted for the treatment of sewage sludge generated from STPs due to its relatively simple operation and control. In comparison with the mesophilic process, the thermophilic AD shows some advantages, such as an acceleration of the biochemical reactions, a greater extent of pathogen reduction and an effective degradation of organic matter. The thermophilic treatment is also increasingly introduced because of the increased demands on sewage sludge treatment. Although in recent researches, Narumiya et al. [4] and Samaras et al. [5] reported the fate and removal of some pharmaceuticals during mesophilic and/or thermophilic ADs in full-scale STPs, there is still very limited information about the variations and removal of PhACs during ADs of sewage sludge.

\section{MATERIALS AND METHODS}

\section{A. Anaerobic Digestion}

Raw sewage sludge used in this work was collected from a STP located in east district of Shanghai, China. The STP was built in 1920s, employing anaerobic digestion processes with one common reactor for the digestion of mixtures of primary and biological (secondary) sewage sludge. Two labscale anaerobic digesters (the total volume of $25 \mathrm{~L}$ for each) were installed in parallel and continuously stirred to keep the homogenous mixture of sewage sludge. The external jackets of the digesters with auto-temperature controller were used to keep their inside temperature. One was operated in the mesophilic range $\left(37^{\circ} \mathrm{C} \pm 2{ }^{\circ} \mathrm{C}\right)$, and the other in the thermophilic range $\left(55^{\circ} \mathrm{C} \pm 2{ }^{\circ} \mathrm{C}\right)$. There were one inlet and two sampling mouths in each digester. The outlet for sludge discharge was set at the bottom of the digester. After condensing, raw sewage sludge which was collected from the sludge circulation lines mentioned above was pumped into the each digester using peristaltic pump. Wet gas flow meter was linked to each digester to count the gas production directly, together with $\mathrm{pH}$ meter monitoring acid-alkaline condition.

After the successful start-up of digesters, they were fed with the sewage sludge previously spiked with the four selected compounds at certain levels. In order to obtain a homogenous spike in the entire volume of sewage sludge, the condensed raw sewage sludge was added with the spikes under continuous stirring $2 \mathrm{~h}$ before its feeding. The settled SRTs, which could be obtained through the gradual adjustment of OLRs, were $10 \mathrm{~d}, 15 \mathrm{~d}$, and $20 \mathrm{~d}$ for the mesophilic anaerobic digester and $7 \mathrm{~d}, 15 \mathrm{~d}$, and $20 \mathrm{~d}$ for the thermophilic condition. The tests were launched after the two digesters ran for two or three SRT periods. Each test 
was done repeatedly. Samples of digested sludge were collected everyday during each test. Each sample was immediately per-treated so as to accurately represent the variations of the target compounds by $\mathrm{AD}$ processes.

\section{B. Sample Pre-treatment and Measurements}

The sewage sludge sample of $500 \mathrm{~mL}$ was pre-filtered with a GF/B glass fiber filter by the vacuum pump. The filtrate with $\mathrm{pH}$ value about 3 adjusted by $\mathrm{H}_{2} \mathrm{SO}_{4}$ addition was introduced to a $\mathrm{C} 8$ cartridges $(6 \mathrm{cc} / 200 \mathrm{mg})$ on a vacuum 12-position Extraction Manifold by means of a PTFE tube for the solid-phase extraction (SPE) to extract the analytes. The biosolid/sludge fraction of the sample retained on the fiber was dried, ground in a mortar, and then pre-treated using ultrasonic solvent extraction. Afterwards, the supernatant was extracted using the same SPE procedure for the aqueous samples.

GC/MS system, Agilent 7890/5975C-GC/MSD, USA, was used for the analysis of target compounds. The parameters for GC/MS described by Zhou et al. [6] were adopted with minor modification. The calculated concentrations of analytes were corrected by the recoveries of the analytes in the samples. The mean recoveries were 64.1\%-82.8\% for CFA, $72.3 \%-82.0 \%$ for DCF, $85.5 \%$ $94.8 \%$ for CMB, and $68.7 \%-90.6 \%$ for TCS. In addition, procedure blanks and solvent blanks were also treated and analyzed alongside with the measurements. The concentrations of the target compounds were calculated with both the aqueous part and the biosolid/sludge fraction of the sewage sludge samples.

\section{RESULTS AND DISCUSSION}

\section{A. Mesophilic Anaerobic Digestion}

When the sludge spiked with $5 \mu \mathrm{g} / \mathrm{L}$ of each selected pharmaceutical was fed into the mesophilic $\mathrm{AD}$, it could be detected in the aqueous phase to some extends. The pharmaceuticals present in the biosolid could, on one hand, be biodegraded, on the other hand, be released into the aqueous phase for the further degradation. At this time, it was hardly to make clear the proportions of the biodegradation in the biosolid/sludge phase in the aqueous phase as well as the desorption from the biosolid. The removal of PhACs for the digested biosolid could be composed of the three parts. Nearly all the concentrations of the selected PhACs in the aqueous were below $0.3 \mu \mathrm{g} / \mathrm{L}$ during the experimental period. In contrast, the selected $\mathrm{PhACs}$ in the biosolid/sludge phase were at 1.0-3.5 $\mu \mathrm{g} / \mathrm{L}$, showing a considerable residue still present in the digested sludge. Also, it implied that adsorption to the biosolid could not be inconsiderable factor during the $\mathrm{AD}$ processes.

According to the selected PhACs in the two phases aforementioned, the overall removal of the compounds during the $\mathrm{AD}$ could be obtained. TABLE I presents the overall removal of all the compounds during the mesophilic $\mathrm{AD}$ at three SRTs. The removal of the selected PhACs went up with the increase of SRTs from $10 \mathrm{~d}$ to $20 \mathrm{~d}$. Especially, TCS demonstrated a distinct trend of the increase, and its overall removal efficiency was raised from $44 \%$ at SRT 10 d to about $74 \%$ at SRT $20 \mathrm{~d}$. The other three compounds also showed the increases on their overall removal efficiencies to certain extents with the extension of SRTs. It could be seen from TABLE I that SRT has a great influence on the removal of the selected PhACs, and the increase on SRT will positively benefit the removal.

\section{B. Thermophilic Anaerobic Digestion}

The thermophilic AD was also operated at three SRTs. The proportions of the selected PhACs in the biosolid/sludge phase and aqueous phase showed a similar variation tendency in this thermophilic mode to the mesophilic mode. However, there were still some differences between the two modes considering the measured data. The overall removal of the four $\mathrm{PhACs}$ during the thermophilic $\mathrm{AD}$ at three SRTs is presented in TABLE I . In general, the removal of the selected PhACs increased with the SRTs from $7 \mathrm{~d}$ to $20 \mathrm{~d}$, however, CFA and TCS showed litter rise when the SRT was set above 15 d. The other two compounds, CMB and DCF, also showed the increases on their overall removal efficiencies with SRTs to certain extents. Notwithstanding, the increases would be slowly down above SRT $15 \mathrm{~d}$. Therefore, SRTs also influenced considerably the removal of the selected PhACs, and the extension on SRTs would be helpful for the removal.

TABLE I. THE OVERALL REMOVAL OF SELECTED PHACS DURING MESOPHILIC AND THERMOPHILIC ADS AT THREE SRTS

\begin{tabular}{|c|c|c|c|c|c|c|c|c|c|}
\hline \multirow{2}{*}{$\begin{array}{c}\text { Anaerobic } \\
\text { digestion }\end{array}$} & \multirow{2}{*}{$\begin{array}{c}\text { SRT } \\
\text { (d) }\end{array}$} & \multicolumn{2}{|c|}{ CFA } & \multicolumn{2}{|c|}{ TCS } & \multicolumn{2}{|c|}{ CBM } & \multicolumn{2}{|c|}{ DCF } \\
\hline & & $\begin{array}{c}\text { Digested } \\
\text { sludge }(\mu g / L) \\
\end{array}$ & $\begin{array}{c}\text { Removal } \\
\text { efficiency }\end{array}$ & $\begin{array}{c}\text { Digested } \\
\text { sludge }(\mu g / L) \\
\end{array}$ & $\begin{array}{c}\text { Removal } \\
\text { efficiency }\end{array}$ & $\begin{array}{c}\text { Digested } \\
\text { sludge }(\mu g / L)\end{array}$ & $\begin{array}{c}\text { Removal } \\
\text { efficiency }\end{array}$ & $\begin{array}{c}\text { Digested } \\
\text { sludge }(\mu g / L) \\
\end{array}$ & $\begin{array}{r}\text { Removal } \\
\text { efficiency }\end{array}$ \\
\hline \multirow{3}{*}{ Mesophilic } & 10 & 2.1 & $57 \%$ & 2.8 & $44 \%$ & 2.5 & $50 \%$ & 2.1 & $59 \%$ \\
\hline & 15 & 1.9 & $61 \%$ & 2.5 & $50 \%$ & 2.3 & $54 \%$ & 1.6 & $67 \%$ \\
\hline & 20 & 1.8 & $63 \%$ & 1.27 & $74 \%$ & 2.0 & $59 \%$ & 1.4 & $71 \%$ \\
\hline \multirow{3}{*}{ Thermophilic } & 7 & 2.2 & $57 \%$ & 2.4 & $52 \%$ & 2.6 & $48 \%$ & 2.4 & $52 \%$ \\
\hline & 15 & 1.7 & $64 \%$ & 1.3 & $74 \%$ & 2.1 & $56 \%$ & 1.9 & $60 \%$ \\
\hline & 20 & 1.6 & $65 \%$ & 1.3 & $74 \%$ & 1.9 & $61 \%$ & 1.8 & $63 \%$ \\
\hline
\end{tabular}




\section{Comparisons of PhACs during Mesophilic and Thermophilic Anaerobic Digestions}

Operating temperature is also an important factor affecting the performances of $\mathrm{AD}$ processes on the removal of the selected PhACs, which could be verified by the above measured data. However, the four targets demonstrated inconsistent influences of the operating temperature during the thermophilic and mesophilic modes. It could be seen from TABLE I that a big increase on the overall removal of TCS from $50 \%$ to the maximal $74 \%$ is obtained during the thermophilic mode at SRT $15 \mathrm{~d}$, compared with the mesophilic mode. The increase on the operating temperature would also benefit the removal of CFA and CBM, showing slight rises at every set SRTs. In contrast, the comparison of the overall removal of DCF in the two modes presented its negative impact of the operating temperature. The thermophilic operation would lead to the perceptible decrease on the removal of DCF during $\mathrm{AD}$ at the set SRTs. The decrease on the DCF removal might be due to the increase on the inhibition of DCF to the related anaerobic bacteria with the rise of the temperature. Therefore, the mesophilic mode seemed to be more favorable to the removal of DCF.

In general, the maximal overall removal was about $65 \%$ for CFA, obtained in the thermophilic mode at SRT $20 \mathrm{~d}$, and up to $74 \%$ for TCS obtained in the thermophilic mode at SRT $15 \mathrm{~d}, 61 \%$ for CBM obtained in the thermophilic mode at SRT $20 \mathrm{~d}$, and $71 \%$ for DCF obtained in the mesophilic mode at SRT $20 \mathrm{~d}$. All the compounds could obtain only the partial removal during the $\mathrm{AD}$ processes, and they might enter into the natural environment when discharged or reused, causing potential environmental risk. Besides, the selected PhACs could be biodegraded into intermediate products [7], which were not detected in this study, and may still cause, even enhance the eco-environmental risks. Therefore, some technical measures are expected to take to enhance the performance of the $\mathrm{AD}$ processes on their removal. Also, further study are being conducted in our lab to analyze the intermediate products and their final removal paths of the compounds during the anaerobic digestion of sewage sludge.

The overall removal efficiency of the selected DCF was comparable to the previous study reported by Carballa et al. [8] in which the mesophilic and thermophilic anaerobic digestions of selected pharmaceutical including DCF and CMB at the different SRTs were investigated. The removal of $\mathrm{DCF}$ during the $\mathrm{AD}$ of sewage sludge was in the range of $69 \% \pm 10 \%$ in that previous study. Besides, DCF under the mesophilic condition could also obtain the relatively higher removal than under the thermophilic condition. However, CMB showed no elimination, differently from its partial removal obtained in this study. The differences between the two studies may be partly attributed to the initial concentrations of the compound in the raw sewage sludge. Much higher-level compound was spiked into the sludge before feeding, possibly leading to some inhibitions to its removal. Another reason might be the source of the raw sewage sludge. In this study, the raw sewage sludge was from the circulation lines of the biological treatment units. The sludge possessed the property of excellent bioactivity and facultative anaerobic bacteria, which could be cultivated into anaerobic activated sludge with no great difficulty, maintaining stable content of bacteria for anaerobic digestion. As for the removal of TCS during AD, there also existed opposite reports. Samaras et al. [9] investigated several PhACs in the two full-scale STPs, Greece, and found the removal rate of TCS using the mesophilic AD was about $52 \%$, and the similar removal rate $(30 \%-50 \%)$ were obtained by Narumiya et al. [10] who conducted the survey of the fate of pharmaceuticals and personal products (PPCPs) including TCS during the mesophilic and thermophilic ADs in the four full-scale STPs. The slightly higher removal efficiency of TCS was got in this study. However, another mesophilic AD experiment using anaerobic soil [11] showed TCS could hardly be removed within $70 \mathrm{~d}$ of the experimental periods. To the best of our knowledge, there has been no report on the behavior of CFA using $\mathrm{AD}$ processes for the time being. Notwithstanding, Salgado et al. [12] studied the biotransformation of CFA in aerobic sequencing batch reactors (SBRs) with mixed microbial cultures, monitoring the efficiency of biotransformation of CFA and the production of metabolites. The maximum removal achieved was $51 \%$. They concluded that CFA is indeed biodegradable.

\section{CONCLUSIONS}

$\mathrm{AD}$ processes operated in the mesophilic and thermophilic modes could partly remove the PhACs from the sludge, however, the overall removal was limited, and their residues and intermediate products in the sludge would still bring potential environmental risks. Therefore, further measures are expected to take to enhance the performances of $\mathrm{AD}$ processes on their removal.

SRTs and operating temperature are two important factors affecting the performances of $\mathrm{AD}$ processes. The increases on SRTs could lead to the rises on the removal of the selected PhACs, and the thermophilic mode would be helpful for the removal of CFA, CBM, and TCS, however, the removal of DCF was found more suitable in the mesophilic mode. Besides, CFA, firstly reported in this study, could obtain maximal $65 \%$ removal efficiency during ADs of sewage sludge.

\section{ACKNOWLEDGEMENTS}

This work was financially supported by the National Natural Science Foundation of China (NSFC) (Grant No. 51279108), the Innovation Program of Shanghai Municipal Education Commission, China (12YZ100), the special fund of State Key Joint Laboratory of Environment Simulation and Pollution Control (12K11ESPCT), and the Major Project of Shanghai Municipal Science and Technology Commission (13DJ1400105).

\section{REFERENCES}

[1] S. Aguayo, A. De La Torre, M. Carballo, R. Cabrera, J. Méndez, "A review of the most relevant residues of pharmaceuticals products of 
human use in effluents and sludges from waste water treatment plants," Toxicol Lett, vol. 196, pp. S65, July 2010.

[2] J. Radjenovic, M. Petrovic, D. Barcelo, "Fate and distribution of pharmaceuticals in wastewater and sewage sludge of the conventional activated sludge (CAS) and advanced membrane bioreactor (MBR) treatment," Water Res ,2009, vol. 43, pp. 831-841.

[3] M. Carballa, F. Omil, T. Ternes, J. M. Lema, "Fate of pharmaceutical and personal care products (PPCPs) during anaerobic digestion of sewage sludge," Water Res, 2007,vol. 41, pp. 2139-2150.

[4] M. Narumiya, N. Nakada, N. Yamashita, H. Tanaka, "Phase distribution and removal of pharmaceuticals and personal care products during anaerobic sludge digestion," J Hazard Mater, 2013, http://dx.doi.org/10.1016/j.jhazmat.2013.05.032:

[5] V. G. Samaras, A. S. Stasinakis, D. Mamais, N. S. Thomaidis, T. D. Lekkas, "Fate of selected pharmaceuticals and synthetic endocrine disrupting compounds during wastewater treatment and sludge anaerobic digestion," J Hazard Mater, 2013, pp. 244-245: 259-267.

[6] H. Zhou, Y. Zhou, H. Li, F. Wang, "Fate and removal of selected endocrine-disrupting compounds in sewage using activated sludge treatment," Water and Environment Journal, 2012, vol. 26, pp. 435444.

[7] A. D. Coelho, C. Sans, A. Agüera, M. J. Gómez, S. Esplugas, M.
Dezotti, "Effects of ozone pre-treatment on diclofenac: Intermediates, biodegradability and toxicity assessment," Sci Total Environ, 2009, vol. 407, pp. 3572-3578.

[8] M. Carballa, F. Omil, T. Ternes, J. M. Lema, "Fate of pharmaceutical and personal care products (PPCPs) during anaerobic digestion of sewage sludge," Water Res, 2007,vol. 41, pp. 2139-2150.

[9] V. G. Samaras, A. S. Stasinakis, D. Mamais, N. S. Thomaidis, T. D. Lekkas, "Fate of selected pharmaceuticals and synthetic endocrine disrupting compounds during wastewater treatment and sludge anaerobic digestion," J Hazard Mater, 2013, pp. 244-245: 259-267.

[10] M. Narumiya, N. Nakada, N. Yamashita, H. Tanaka, "Phase distribution and removal of pharmaceuticals and personal care products during anaerobic sludge digestion," J Hazard Mater, 2013, http://dx.doi.org/10.1016/j.jhazmat.2013.05.032:

[11] G. Ying, X. Yu, R. S. Kookan, "Biological degradation of triclocarban and triclosan in a soil under aerobic and anaerobic conditions and comparison with environmental fate modelling," Environ Pollut, 2007, vol.15, pp. 300-305.

[12] R. Salgado, A. Oehmen, G. Carvalho, J. P. Noronha, M. A. Reis, "Biodegradation of clofibric acid and identification of its metabolites," J Hazard Mater, 2012, pp. 241-242: 182-189. 